\title{
ARTICLES
}

\section{The virtual origin as a first-order correction for the far-field description of laminar jets}

\author{
Antonio Revuelta and Antonio L. Sánchez \\ Area de Mecánica de Fluidos, Departamento de Ingeniería Mecánica, Universidad Carlos III de Madrid, \\ 28911 Leganés, Spain \\ Amable Liñán \\ Departamento de Motopropulsión y Termofluidodinámica, ETSI Aeronáuticos, Universidad Politécnica de \\ Madrid, 28040 Madrid, Spain
}

(Received 9 November 2001; accepted 7 March 2002; published 26 April 2002)

\begin{abstract}
The far-field velocity and composition fields of a submerged laminar jet are known to approach a self-similar solution corresponding to the flow induced by a point source of momentum and scalar. Previous efforts to improve this far-field description have introduced a virtual origin for the streamwise coordinate to remedy the singular behavior of the self-similar solution near the jet origin. The purpose of this note is to show, by means of a perturbative analysis of the point-source solution, that this virtual origin is in fact the first-order correction to the leading-order description. The perturbative analysis, which uses the ratio $x$ of the streamwise distance to the length of jet development as an asymptotically large quantity, also indicates that the displaced point source provides the description in the far field with small relative errors of order $x^{-3}$ for the round jet and of order $x^{-10 / 3}$ for the plane jet. The values of the virtual origin are obtained by numerical integration of the boundary-layer equations in the region of jet development, giving values that depend on the shape of the jet velocity profile at the exit. (C) 2002 American Institute of Physics.
\end{abstract} [DOI: $10.1063 / 1.1473650]$

The structure of a submerged jet is known to depend on the value of its associated jet Reynolds number Re. For moderately large values of $R e$ the resulting steady laminar jet remains stable, and can be calculated with errors of order $R e^{-2}$ by integrating the boundary-layer form of the conservation equations. The jet is initially separated from the outer stagnant flow by an annular mixing layer that grows from the injector rim. At distances of the order of $R e$ times the initial transverse dimension of the jet the effect of viscosity starts to reduce significantly the value of the velocity at the axis. As shown by Schlichting for the round jet ${ }^{1}$ and by Bickley ${ }^{2}$ for the planar jet, the flow downstream from this development region approaches a self-similar solution corresponding to the flow induced by a point source of momentum.

These asymptotic solutions were verified experimentally by Andrade and Tsien ${ }^{3}$ and Andrade, ${ }^{4}$ who measured the distribution of velocity in a liquid-into-liquid jet. They found that the accuracy of the point-source solution can be improved by displacing the location of the point source upstream from the jet exit.

The present short communication is intended to contribute further understanding of the far-field description of laminar jets at $R e \gg 1$. The study considers not only the velocity field but also the mixing process of a passive scalar carried by the jet with the surrounding stagnant fluid. ${ }^{5}$ The distance from the jet exit scaled with the length of jet development, $x$, will be used as an asymptotically large quantity to construct the solution in the far field. Investigation of the eigensolutions of the asymptotic descriptions of Schlichting and Bickley $^{1,2}$ will reveal that the first-order correction for the velocity and composition field is indeed equivalent to the introduction of a displaced origin for the point source of momentum and scalar, as originally postulated by Andrade and Tsien $^{3}$ and Andrade. ${ }^{4}$ We shall see that, with an appropriate selection for the point-source location, $x=x_{o}$, the resulting solution describes the velocity and composition in the far field with small relative errors that are of order $x^{-3}$ for the round jet and of order $x^{-10 / 3}$ for the plane jet. The value of $x_{o}$, which depends only on the shape of the jet velocity profile at the inlet, will be determined by numerical integration of the conservation equations in the region of jet development.

We consider the submerged jet formed when an incompressible fluid of density $\rho$ and kinematic viscosity $\nu$ flows into an unconfined space filled with the same fluid. When the Reynolds number $R e=U_{o} a / \nu$, based on the characteristic exit velocity $U_{o}$ and on the characteristic transverse dimension $a$ (the initial radius for the round jet and the initial half width for the planar jet), is moderately large, then the resulting jet is steady and slender. The solution can be computed with the boundary-layer approximation by integrating numerically the equations 


$$
\begin{aligned}
& \frac{\partial u}{\partial x}+\frac{1}{r^{j}} \frac{\partial\left(r^{j} v\right)}{\partial r}=0, \\
& u \frac{\partial u}{\partial x}+v \frac{\partial u}{\partial r}=\frac{1}{r^{j}} \frac{\partial}{\partial r}\left(r^{j} \frac{\partial u}{\partial r}\right), \\
& u \frac{\partial Y}{\partial x}+v \frac{\partial Y}{\partial r}=\frac{1}{S} \frac{1}{r^{j}} \frac{\partial}{\partial r}\left(r^{j} \frac{\partial Y}{\partial r}\right),
\end{aligned}
$$

with boundary conditions

$$
\begin{aligned}
& r=0: \frac{\partial u}{\partial r}=v=\frac{\partial Y}{\partial r}=0, \\
& r \rightarrow \infty: u=Y=0 .
\end{aligned}
$$

and with initial conditions at $x=0$

$$
\begin{aligned}
& 0 \leqslant r \leqslant 1: u-u_{i}(r)=Y-1=0, \\
& r>1: \quad u=Y=0 .
\end{aligned}
$$

The index $j$ takes the value $j=0$ for the planar configuration and $j=1$ for the round jet. The spatial coordinates and the velocity components are scaled with their characteristic values in the region of jet development, leading to a formulation independent of the Reynolds number. Thus, $x$ and $r$ are the streamwise and transverse coordinates scaled with $R e a$ and $a$, respectively, and the axial and radial velocity components $u$ and $v$ are scaled with $U_{0}=\left[J /\left(\rho 2^{1-j} \pi^{j} a^{1+j}\right)\right]^{1 / 2}$ and $\nu / a$, with $J$ denoting here the momentum flux of the jet. The initial velocity distribution $u_{i}(r)$ at $x=0$ depends on the shape of the jet velocity profile. Cases of interest include that of a uniform profile $\left(u_{i}=1\right)$ and that of a fully developed Poiseuille profile $\left(u_{i}=\sqrt{15 / 8}\left(1-r^{2}\right)\right.$ for $j=0$ and $u_{i}$ $=\sqrt{3}\left(1-r^{2}\right)$ for $\left.j=1\right)$. In (3), $S$ represents the transport Schmidt number for the passive scalar.

Radial integration of (2) and (3) yields the integral conservation laws for momentum and scalar flux

$$
\int_{0}^{\infty} 2^{j} r^{j} u^{2} \mathrm{~d} r=\frac{1}{q} \int_{0}^{\infty} 2^{j} r^{j} u Y \mathrm{~d} r=1,
$$

which are satisfied by the solution of (1)-(3). In the formulation, the constant $q=\int_{0}^{1} 2^{j} r^{j} u_{i} \mathrm{~d} r$ is proportional to the initial volume flux, becoming $q=1$ for the initially uniform velocity profile and $q=\sqrt{5 / 6}(j=0)$ and $q=\sqrt{3} / 2(j=1)$ for Poiseuille flow.

The solution to (1)-(5) far downstream from the region of jet development, i.e., for $x \gg 1$, becomes self-similar, and corresponds at leading order to that generated by a pointsource of momentum and scalar located at $x=0 .{ }^{1,2,5}$ For the round jet, ${ }^{1,5}$ where the appropriate self-similar coordinate is $\eta=r / x$, the description of the velocity and composition fields requires the introduction of the stream function $\psi$ $=x F_{0}(\eta)$ and of the scalar concentration $Y=x^{-1} Y_{0}(\eta)$ into (1)-(4) to give $F_{0}^{\prime \prime \prime}+F_{0}^{\prime \prime}\left(F_{0}-1\right) / \eta+F_{0}^{\prime}\left(1-F_{0}+\eta F_{0}^{\prime}\right) / \eta^{2}$ $=0 ; F_{0}(0)=\left(F_{0}^{\prime} / \eta\right)^{\prime}(0)=F_{0}^{\prime}(\infty)=0$ and $\left(\eta Y_{0}^{\prime}+S F_{0} Y_{0}\right)^{\prime}$ $=0 ; Y_{0}^{\prime}(0)=Y_{0}(\infty)=0$. Integrating the above problems with the additional constraints $2 \int_{0}^{\infty} F_{0}^{\prime 2} / \eta \mathrm{d} \eta=(2 / q) \int_{0}^{\infty} F_{0}^{\prime} Y_{0} \mathrm{~d} \eta$ $=1$ arising from (6) yields ${ }^{1,5}$

$$
F_{0}=\frac{4 \eta^{2}}{64 / 3+\eta^{2}} \text { and } Y_{0}=\frac{(2 S+1) q / 8}{\left(1+3 \eta^{2} / 64\right)^{2 S}}
$$

This Schlichting-Squire solution $\psi=x F_{0}(\eta)$ and $Y$ $=x^{-1} Y_{0}(\eta)$ is in fact an exact solution for the boundarylayer problem (1)-(4), in which the initial conditions (5) have been replaced by a Dirac-delta function for the velocity and concentration with a strength given by the integral conditions (6). The solution is not unique: since the problem (1)-(4) is translationally invariant, the alternative far-field description

$$
\begin{aligned}
& \psi=\left(x-x_{o}\right) F_{0}\left(\frac{r}{x-x_{o}}\right), \\
& Y=\frac{1}{x-x_{o}} Y_{0}\left(\frac{r}{x-x_{o}}\right),
\end{aligned}
$$

corresponding to a point source located at $x=x_{o}$, is also an exact solution of the boundary-layer problem. Expanding (8) for $x \gg 1$ with use made of the original self-similar coordinate $\quad \eta=r / x \quad$ yields $\quad \psi=x F_{0}(\eta)-x_{o}\left[F_{0}(\eta)-\eta F_{0}^{\prime}(\eta)\right]$ $+O\left(x^{-1}\right)$ and $Y=x^{-1} Y_{0}(\eta)+x^{-2} x_{o}\left[Y_{0}(\eta)+\eta Y_{0}^{\prime}(\eta)\right]$ $+O\left(x^{-3}\right)$, indicating that the displacement $x_{o}$, to be calculated, amounts to a relative correction of order $x^{-1}$ to the far-field Schlichting-Squire solution.

An improved description for the far field requires consideration of perturbations to the point-source solution. Since Schlichting-Squire solution $\left[x F_{0}, x^{-1} Y_{0}\right]$ is an exact solution to (1)-(4), the first-order correction must be necessarily an eigensolution of the problem. One therefore seeks solutions to (1)-(4) obtained by adding an eigensolution of the form $C_{m}\left[x^{1-\lambda_{m}} F_{m}, x^{-1-\lambda_{m}} Y_{m}\right]$ to Schlichting-Squire solution, where $C_{m}$ is an arbitrary constant. ${ }^{6}$ The eigenvalues $\lambda_{m}$ and eigenfunctions $\left[F_{m}(\eta), Y_{m}(\eta)\right]$ are obtained by solving the linear perturbation equations

$$
\begin{aligned}
F_{m}^{\prime \prime \prime}+ & F_{m}^{\prime \prime}\left(F_{0}-1\right) / \eta+F_{m}^{\prime}\left[1-F_{0}+\left(2+\lambda_{m}\right) \eta F_{0}^{\prime}\right] / \eta^{2} \\
& +\left(1-\lambda_{m}\right) F_{m}\left(\eta F_{0}^{\prime \prime}-F_{0}^{\prime}\right) / \eta^{2}=0
\end{aligned}
$$

and

$$
\begin{gathered}
\left(\eta Y_{m}^{\prime}\right)^{\prime} / S+F_{0} Y_{m}^{\prime}+\left(1+\lambda_{m}\right) F_{0}^{\prime} Y_{m} \\
=-Y_{0} F_{m}^{\prime}-\left(1-\lambda_{m}\right) Y_{0}^{\prime} F_{m},
\end{gathered}
$$

obtained from (1)-(3) with the homogeneous boundary conditions $F_{m}(0)=\left(F_{m}^{\prime} / \eta\right)^{\prime}(0)=F_{m}^{\prime}(\infty)=Y_{m}^{\prime}(0)=Y_{m}(\infty)=0$ obtained from (4). Nontrivial solutions to (9) and (10) can be found for an infinite discrete set of eigenvalues $\lambda_{m}=m(m$ $+1) / 2=1,3,6,10,15, \ldots$. Note that once the value of $\lambda_{m}$ and the function $F_{m}$ are determined by integrating numerically (9), the accompanying function $Y_{m}$ is determined uniquely as the particular solution to $(10)$. The first eigensolution $\left[F_{0}\right.$ $\left.-\eta F_{0}^{\prime},-x^{-2}\left(Y_{0}+\eta Y_{0}^{\prime}\right)\right]$, which satisfies identically the integral constraints $\int_{0}^{\infty} F_{0}^{\prime} F_{1}^{\prime} / \eta \mathrm{d} \eta=\int_{0}^{\infty}\left(F_{0}^{\prime} Y_{1}+F_{1}^{\prime} Y_{0}\right) \mathrm{d} \eta=0$ obtained from (6), is the $x$-derivative to the SchlichtingSquire functions. Comparison with the expansions given in (8) indicates that this first eigensolution corresponds to an 
uncertainty in the location of the origin, with the value of $C_{1}=-x_{o}$ representing the unknown upstream displacement of the point source.

The same uncertainty appears in general in any downstream expansion for a boundary-layer flow when the initial conditions are not imposed. ${ }^{6}$ For instance, for the Blasius boundary layer over a flat plate, whose eigenproblem was solved numerically by Libby, ${ }^{7}$ the value of $x_{o}$ is of the order of the characteristic length of the Navier-Stokes region at the plate leading edge. For the laminar jet, the displacement of the point source is of the order of the characteristic length of jet development, giving a value of $x_{o}$ of order unity that can be easily incorporated to provide (8) for the corrected far-field description. Note that, since this is also an exact solution of (1)-(4), its perturbations must be associated with the second eigenvalue $\lambda_{2}=3$ obtained from (9). Therefore, with the apropriate choice for the virtual origin, equations (8) give the boundary-layer far-field solution for the jet with small relative errors of order $x^{-3}$. Note that the effect of streamwise diffusion, which is neglected in the boundarylayer approximation, causes perturbations to the axial velocity of order $x^{-1} R e^{-2}$. Sufficiently far downstream, these perturbations become larger than the departures from similarity associated with the second eigenvalue, $\lambda_{2}=3$, which give perturbations to the axial velocity of order $x^{-4}$.

The asymptotic solution for the planar jet at $x \gg 1$ parallels that given above for the round jet. In this case $(j=0)$, the appropriate selfsimilar coordinate is $\zeta=\alpha r / x^{2 / 3}{ }^{2}$, where the numerical coefficient $\alpha=1 /\left(2 \cdot 3^{1 / 3}\right)$ is introduced to simplify the notation. The selfsimilar stream function $\psi$ $=x^{1 / 3} f_{0}(\zeta)^{2}$ and scalar $Y=x^{-1 / 3} y_{0}(\zeta)$ must be employed in this case, where the functions

$$
f_{0}=6 \alpha \tanh \zeta \text { and } y_{0}=\frac{q \operatorname{sech}^{2 S} \zeta}{3 \alpha \mathrm{B}\left(S+1, \frac{1}{2}\right)},
$$

are determined by integrating $\left(3 \alpha f_{0}^{\prime \prime}+f_{0} f_{0}^{\prime}\right)^{\prime}=0 ; f_{0}(0)$ $=f_{0}^{\prime \prime}(0)=f_{0}^{\prime}(\infty)=0 \quad$ and $\quad\left(3 \alpha y_{0}^{\prime} / S+f_{0} y_{0}\right)^{\prime}=0 ; \quad y_{0}^{\prime}(0)$ $=y_{0}(\infty)=0$, obtained from (1)-(4), subject to the additional constraints $\int_{0}^{\infty} \alpha f_{0}^{\prime 2} \mathrm{~d} \zeta=q^{-1} \int_{0}^{\infty} y_{0} f_{0}^{\prime} \mathrm{d} \zeta=1$ emerging from (6). Here, the prime denotes differentiation with respect to the similarity coordinate $\zeta$ and $B()$ represents the Beta function.

To seek a correction to the Bickley asymptotic description $\left[x^{1 / 3} f_{0}, x^{-1 / 3} y_{0}\right]$, which is an exact solution to (1)-(4), one considers perturbations of the form $C_{m}\left[x^{1 / 3-\lambda_{m}} f_{m}, x^{-1 / 3-\lambda_{m}} y_{m}\right]$, a development that leads to the linear equations

$$
\alpha f_{m}^{\prime \prime \prime}+\frac{1}{3} f_{0} f_{m}^{\prime \prime}+\left(\frac{2}{3}+\lambda_{m}\right) f_{0}^{\prime} f_{m}^{\prime}+\left(\frac{1}{3}-\lambda_{m}\right) f_{0}^{\prime \prime} f_{m}=0
$$

and

$$
\begin{gathered}
\alpha y_{m}^{\prime \prime} / S+\frac{1}{3} f_{0} y_{m}^{\prime}+\left(\frac{1}{3}+\lambda_{m}\right) f_{0}^{\prime} y_{m} \\
=-\left(\frac{1}{3}-\lambda_{m}\right) y_{0}^{\prime} f_{m}-y_{0} f_{m}^{\prime},
\end{gathered}
$$

to be solved with boundary conditions $f_{m}(0)=f_{m}^{\prime \prime}(0)$ $=f_{m}^{\prime}(\infty)=y_{m}^{\prime}(0)=y_{m}(\infty)=0$. It is worth pointing out that the same eigenvalue problem arises in the study of perturbations to the self-similar radial jet. The solution was deter-
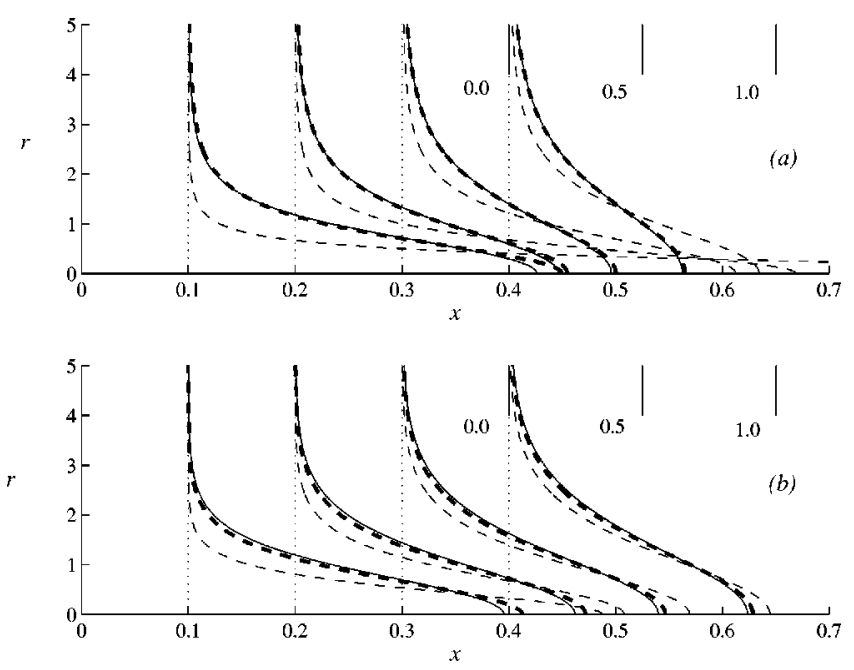

FIG. 1. The velocity profiles for the planar jet (a) and for the round jet (b) as obtained from numerical integration of (1)-(5) (solid lines), from the solution of the point source at $x=0$ (thin dashed lines), and from the solution of the point source at $x=x_{o}$ (thick dashed lines); the scale for the velocity is indicated above the plots corresponding to $x=0.4$.

mined analytically by Riley, ${ }^{8}$ who found that nontrivial solutions exist for $\lambda_{m}=m(2 m+1) / 3=1,10 / 3,21 / 3,12,55 / 3, \ldots$, with the first eigenfunction $\left[x^{-2 / 3}\left(f_{0}-2 \zeta f_{0}^{\prime}\right) / 3, x^{-4 / 3}\left(y_{0}\right.\right.$ $\left.+2 \zeta y_{0}^{\prime}\right) / 3$ ] being the $x$-derivative of Bickley solution. As before, this first correction, which satisfies the integral conditions $\int_{0}^{\infty} f_{0}^{\prime} f_{1}^{\prime} \mathrm{d} \zeta=\int_{0}^{\infty}\left(f_{0}^{\prime} y_{1}+f_{1}^{\prime} y_{0}\right) \mathrm{d} \zeta=0$ arising from (6), is due to an upstream displacement $C_{1}=-x_{o}$ of the origin, and reflects the fact that the alternative solution

$$
\begin{aligned}
& \psi=\left(x-x_{o}\right)^{1 / 3} f_{0}\left(\frac{\alpha r}{\left(x-x_{o}\right)^{2 / 3}}\right), \\
& Y=\frac{1}{\left(x-x_{o}\right)^{1 / 3}} y_{0}\left(\frac{\alpha r}{\left(x-x_{o}\right)^{2 / 3}}\right),
\end{aligned}
$$

corresponding to a point source located at $x=x_{o}$, is also an exact solution of (1)-(4). Since perturbations to (14) can only be associated with the second eigensolution, the relative error of this modified solution can be expected to be of order $x^{-10 / 3}$.

The asymptotic analysis presented above does not determine the value of $x_{o}$, which depends on the development of the jet in the region $x \sim 1$ corresponding to distances of order Re times $a$ from the jet exit. The value of $x_{o}$ can be calculated from $x_{o}=\lim _{x \rightarrow \infty}\left[x-3 /\left(8 u_{\max }\right)\right]$ (round jet) and $x_{o}$ $=\lim _{x \rightarrow \infty}\left[x-\left(6 \alpha^{2} / u_{\max }\right)^{3}\right]$ (planar jet), where $u_{\max }$ is the velocity along the axis calculated from the numerical integrations of (1)-(5). The result depends on the shape of the initial velocity profile $u_{i}(r)$, e.g., $x_{o}=-0.230$ (round) and $x_{o}$ $=-0.190$ (planar) for $u_{i}=1$ and $x_{o}=-0.168$ (round) and $x_{o}=-0.105$ (planar) for a parabolic profile. Note that the results for the round jet are in close agreement with the values $x_{o}=-0.225$ (uniform) and $x_{o}=-0.173$ (parabolic) obtained by Andrade and Tsien ${ }^{3}$ by imposing that the resulting kinetic-energy flux of the point-source solution at the jet exit be equal to that of the initial jet velocity profile. The same criterion applied to the planar jet also yields accurate values 

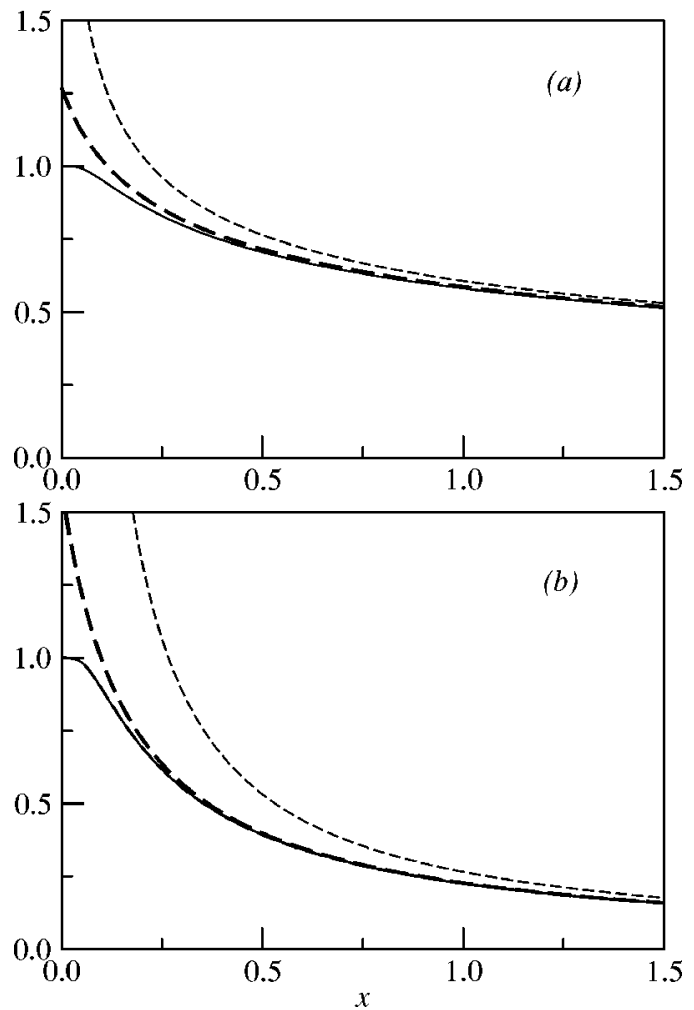

FIG. 2. The evolution of $Y$ along the axis for the planar jet (a) and for the round jet (b) as obtained with an initial parabolic profile from numerical integration of (1)-(5) (solid lines), from the solution of the point source at $x=0$ (thin dashed lines), and from the solution of the point source at $x$ $=x_{o}$ (thick dashed lines).

for $x_{o}$; e.g., $x_{o}=-0.192$ for $u_{i}=1$ and $x_{o}=-0.119$ for $u_{i}$ $=\sqrt{15 / 8}\left(1-r^{2}\right)$. The degree of coincidence suggests that the viscous dissipation in the development region does not differ much from that predicted by point-source solution, so that correcting the kinetic-energy flux at the jet exit suffices to calculate approximately the far-field correction.

It is worth noting that the selection of the virtual origin for the round jet corresponds to a correction of order unity in the point-source volume flux. ${ }^{9}$ To see this, one may integrate (1) to obtain

$$
\int_{0}^{\infty} 2 \pi r u \mathrm{~d} r=\pi q+\int_{0}^{x} \Phi\left(x^{\prime}\right) \mathrm{d} x^{\prime},
$$

indicating that the volume driven by the jet increases from the initial value $\pi q$ due to additional fluid that is being entrained with a rate $\Phi(x)=-(2 \pi r v)_{r \rightarrow \infty}$, a function calcu- lated in Ref. 9 which decreases for increasing values of $x$ towards the constant asymptotic value $\Phi=8 \pi$ of Schlichting solution. ${ }^{1}$ According to (8), at distances $x \gg 1$ the volume flux becomes $\int_{0}^{\infty} 2 \pi r u \mathrm{~d} r=2 \pi F_{0}(\infty)\left(x-x_{o}\right)=8 \pi\left(x-x_{o}\right)$. Clearly, the volume flux of Schlichting solution, $\int_{0}^{\infty} 2 \pi r u \mathrm{~d} r$ $=8 \pi x$, accounts only for the fluid that has been entrained with the constant asymptotic rate $\Phi=8 \pi$. The virtual origin

$$
x_{o}=-\left[\int_{0}^{\infty}[\Phi /(8 \pi)-1] \mathrm{d} x+q / 8\right] \text {, }
$$

obtained from (15) at the following order, corrects this leading-order result by accounting for the initial volume flux as well as for the larger entrainment rate $\Phi>8 \pi$ that occurs in the region of jet development $x \sim 1$.

The asymptotic results given in (8) and (14) are compared in Figs. 1 and 2 with results of numerical integrations of (1)-(5). Velocity profiles across the jet obtained with an initial Poiseuille profile at the jet exit are shown in Fig. 1. The plot indicates that the virtual origin improves considerably the leading-order results, so that close agreement with the numerical integrations is observed already at distances $x=0.1$. The same level of accuracy is obtained for the description of the mixing process, as can be seen in Fig. 2, where the evolution of the scalar $Y$ along the axis is plotted for $S=0.73$, as corresponds to the temperature evolution in an air jet. It is remarkable that the virtual origin is the same for the velocity and for the scalar, independently of the scalar diffusivity.

\section{ACKNOWLEDGMENTS}

Useful discussions with Norman Riley are gratefully acknowledged. This work was supported by the Spanish DGESIC under Project \#PB98-0142-C04-02 and by the Spanish MCYT under Project \#BFM2001-3691.

${ }^{1}$ H. Schlichting, "Laminare strahlenausbreitung," Z. Angew. Math. Mech. 13, 260 (1933).

${ }^{2}$ W. Bickley, "The plane jet," Philos. Mag. 23, 727 (1937).

${ }^{3}$ E. N. Andrade and L. C. Tsien, "The velocity distribution in a liquid into liquid jet," Proc. Phil. Soc. London 49, 381 (1937).

${ }^{4}$ E. N. Andrade, "The velocity distribution in a liquid into liquid jet. The plane jet," Proc. Phil. Soc. London 51, 784 (1939).

${ }^{5}$ H. B. Squire, "The round laminar jet," Q. J. Mech. Appl. Math. 4, 321 (1951).

${ }^{6} \mathrm{~K}$. Stewartson, "On asymptotic expansions in the theory of boundary layers,” J. Math. Phys. (Cambridge, Mass.) 36, 173 (1957).

${ }^{7}$ P. A. Libby, "Eigenvalues and norms arising in perturbations about the Blasius solution," AIAA J. 3, 2164 (1965).

${ }^{8}$ N. Riley, "Asymptotic expansions in radial jets," J. Math. Phys. 41, 132 (1962).

${ }^{9}$ A. Revuelta, A. L. Sánchez, and A. Liñán, “Confined axisymmetric laminar jets with large expansion ratios," J. Fluid Mech. 456, 319 (2002). 
Physics of Fluids is copyrighted by the American Institute of Physics (AIP). Redistribution of journal material is subject to the AIP online journal license and/or AIP copyright. For more information, see http:/ojps.aip.org/phf/phfcr.jsp

Copyright of Physics of Fluids is the property of American Institute of Physics and its content may not be copied or emailed to multiple sites or posted to a listserv without the copyright holder's express written permission. However, users may print, download, or email articles for individual use. 